\title{
Grenzenlose Biografien und ihr begrenzter (Bildungs-)Erfolg. Das Thema der sozialen Ungleichheit aus der Perspektive eines laufenden soziolinguistischen Forschungsprojekts
}

\begin{abstract}
Das hier vorgestellte, zurzeit noch laufende Forschungsprojekt („Best success through Language Loss?", ${ }_{1}$ 2008-2012/13) hat es sich zum Ziel gesetzt, dem traditionell soziologischen Thema der sozialen Ungleichheit aus linguistischer Perspektive nachzuspüren. Das Phänomen von zentralem Interesse sind die wiederkehrend ungleichen Bildungserfolge verschiedener, gerade auch migrantischer Bevölkerungsgruppen in Europa, wobei die Untersuchung ein Sample von rund 180 Kindern unterschiedlichster sozialer und migrantischer Herkunft in Wien im Fokus hat. Das Thema erfordert für die Methodik auf allen Ebenen vor allem interdisziplinäre Offenheit. Der vorliegende Artikel wird beschreiben, wie die Untersuchung diesem Anspruch gerecht zu werden versucht.
\end{abstract}

\section{Einleitung}

Die hier vorzustellende Untersuchung, bewilligt und finanziert durch den österreichischen Forschungsfonds FWF, startete im Jahr $2008^{2}$ und befindet sich zum Zeitpunkt des Verfassens dieses Artikels (Sommer 2012) in ihrem letzten Laufjahr. Angesiedelt ist die Untersuchung an der Österreichischen Akademie der Wissenschaften in Wien, mit kooperierenden Institutionen in Österreich, Deutschland, den Niederlanden und der Türkei.

Da es sich um ein laufendes Forschungsprojekt handelt, liegen Ergebnisse noch nicht oder nur ansatzweise vor. Dieser Artikel wird sich daher vor allem mit der Idee und Zielsetzung befassen, die hinter dem Gesamtvorhaben steht, das heißt: mit den Beweggründen für die Untersuchung (Abschnitt 2), mit dem theoretischen Rahmen (Abschnitt 3) und mit der Sample- und Datengenerierung sowie der Methodik insgesamt (Abschnitt 4). Erste Analysen werden hingegen nur beispielhaft skizziert (Abschnitt 5), abgerundet durch abschließende Bemerkungen zum Gesamtprojekt (Abschnitt 6).

Österreichischer Forschungsfonds FWF, Projektnummer P20263-G03.

Die Laufzeit beträgt drei Jahre, mit der Möglichkeit der kostenneutralen Verlängerung um max. zwei weitere Jahre. 


\section{Beweggründe für die Untersuchung}

Wiewohl ein linguistisches Unterfangen, widmet sich die hier vorgestellte Untersuchung ganz zentral dem Begriff der sozialen Ungleichbeit, und damit also einem Kernthema nicht eigentlich der Linguistik, sondern der Soziologie: Diese definiert soziale Ungleichheit als dann gegeben,

[...] wenn Menschen aufgrund ihrer Stellung in sozialen Beziehungsgefügen von den „wertvollen Gütern“ einer Gesellschaft regelmäßig mehr als andere erhalten. (Hradil/Schiener 2001, S. 30)

Die vielfältigen Gründe dafür, dass dieses soziologische Kernthema zum Leitfaden auch einer linguistischen Untersuchung wird, möchte ich hier in drei Schritten zusammenfassen.

Erstens manifestieren sich Phänomene sozialer Ungleichheit überaus häufig gerade auch sprachlich; dies trifft besonders auf die Bildungssysteme moderner Migrationsgesellschaften zu: Schulerfolg definiert sich hier - unter anderem - sehr klar auch als Schulspracherfolg (im Fall von Österreich: als erfolgreiches Abschneiden in der Schulsprache ${ }^{3}$ Deutsch). Ein Beispiel, das für unseren Zusammenhang besonders interessant ist, mag den Zusammenhang verdeutlichen: Die Forschungsliteratur belegt hartnäckig wiederkehrende schulische Misserfolge gerade für bestimmte Herkunftsgruppen in der Migration; betroffen sind vor allem Schülerinnen und Schüler türkischer, marokkanischer und bengalischer Herkunft in Europa (Esser 2006; Maas/Mehlem 2002; Haque 2000 u.v.m.). Das „schwache Abschneiden“, das hier diagnostiziert wird, zeigt sich aber eben nicht nur hinsichtlich Schulerfolg (vgl. z.B. Tilmatine 1997; Herzog-Punzenberger 2003) und Arbeitsmarkt (Esser 2006; Weiss et al. 2007, S. 11), sondern gerade auch bereits in Bezug auf den Erwerb der jeweiligen Schulsprache (vgl. z.B. Rüesch 1998; PeltzerKarpf et al. 2006). Die institutionelle Zuteilung von Schulerfolg - und damit von sozialem Aufstieg - scheint also eng daran geknüpft zu sein, wie sich die sprachlichen Leistungen der Schülerinnen und Schüler im schulischen Kontext äußern, wie sie wahrgenommen und beurteilt werden.

Zweitens stehen sprachliche Manifestationen sozialer Ungleichheit häufig in unmittelbarer Verbindung zu einer weiteren Erscheinungsform von Ungleichheit. Diese lässt sich am einfachsten mit dem Adjektiv ethnisch charakterisieren - inklusive aller problematischen Implikationen dieses Begriffs. Schon das oben genannte Beispiel der „schwachen Gruppen“ führt dies vor Augen. Es sei hier noch ergänzt um ein scheinbar ganz besonders , herkunftsspezifisches" Forschungsergebnis: Zusätzlich zum genannten Abschneiden auf dem Arbeitsmarkt, im Schulerfolg und in der Schulsprache kann sich noch eine weitere, noch schwieriger zu verstehende Ungleichheit zei-

Der Begriff ,Schulsprache‘ bezeichnet hier jene Sprache, in der der Schulunterricht im jeweiligen Einwanderungsland stattfindet. 
gen, und zwar im Bereich der Familiensprachen (vgl. z.B. Peltzer-Karpf et al. $2006^{4}$ ). Für augenscheinlich ,ethnische“ Ungleichheiten gibt es also zahlreiche Belege auf den unterschiedlichsten Ebenen; die Ursachensuche bleibt jedoch schwierig. Denn schneiden soziale, gar „ethnisch“ oder „national“ definierte Gruppen gleichsam „kollektiv“ schwach ab, so drängen leicht Erklärungen in den Vordergrund, die die Ursachen innerhalb der Gruppen selbst lokalisieren - und damit die Problematik „ethnisieren“. Faktoren wie Intelligenz und Selbstvertrauen gehören hierher, ebenso wie familiäre Beziehungsqualitäten, Motivationen u.v.m. (vgl. z.B. Esser 2006): Es sind dies allesamt Erklärungen, die sich für den individuellen Sprachlern- und Schulerfolg als geeignet erwiesen haben, kaum aber großräumig und „kollektiv“, also entlang „,nationaler“ oder „ethnischer“ Grenzen verteilt sein können. Ähnlich verhält es sich darüber hinaus auch mit dem Konzept der „Kultur“, die als Erklärung gerade für Spracherwerbsmisserfolge schlechterdings nicht in Frage kommt; wäre es doch wenig plausibel anzunehmen, dass manche Kulturen den Spracherwerb schlechter bewerkstelligen als andere, zumal den Erwerb der gruppen,internen" Sprachen. Moderne Ungleichheitstheorien gehen daher davon aus, dass Kultur nicht ursächlich formt, sondern zunächst geformt wird, kollektive Phänomene also nicht nur hervorruft, sondern vor allem selbst zu ihnen gehört; Kultur ist damit weniger eine Ursache als vielmehr eine „Folge zugrundeliegender kollektiver (Lebens)Bedingungen" (Hradil/Schiener 2001, S. 89). Indem sie der Kulturalisierung von Wirkungszusammenhängen entgegenstehen, leisten die genannten Ansätze dasselbe auch entgegen einer Ethnisierung: Wie Kulturen, so gehen auch „ethnisch“ oder sprachlich definierte Gemeinschaften zunächst selbst aus materiell, sozial, politisch u.s.w. ungleichen Lebensbedingungen hervor. Sprachliche und ethnische Ungleichheit oder Schichtung (z.B. im Bildungswesen) sind damit als sichtbare Ausprägungen tieferliegender Mechanismen von Ungleichheit zu verstehen (Gomolla/Radtke 2007).

Der dritte Beweggrund schließlich für unseren Fokus auf sozialer Ungleichheit verbindet die beiden bisher besprochenen Beweggründe miteinander: Die vielen Zusammenhänge zwischen spracblicher und ethnischer sozialer Ungleichheit bedürfen, um besser verstanden zu werden, auch einer engeren Verbindung der mit ihnen befassten Forschungsdisziplinen, und damit auch einer linguistischen Ungleichheitsforschung. Gerade dass sich soziale Ungleichheit, insbesondere ethnische Schichtung, auch sprachlich manifestiert, eröffnet ja nicht nur soziologische, sondern zusätzlich vor allem linguistische Wege der Untersuchung. Lange haben sich dennoch Studien zu diesen verwandten Themen in voneinander getrennten Bereichen bewegt. Dies hatte beispielsweise zur Folge, dass „herkunftsspezifische“ Phänomene etwa innerhalb der Psycholinguistik (vgl. Peltzer-Karpf et al. 2006,

Zur kritischen Reflexion dieser Studie siehe Brizić (2009). 
besprochen in Brizić 2009) oder innerhalb der Soziologie (Esser 2006, S. 547) nur schwer interpretierbar waren; Fragen nach den Ursachen für diese Phänomene mussten bisher somit weitgehend offen bleiben (Stanat 2003; Söhn/Özcan 2006).

Das Hauptanliegen der hier vorzustellenden Untersuchung ist es deshalb, beide Disziplinen auf möglichst vielen Ebenen, d.h. inhaltlich-theoretisch wie auch methodisch, zu verbinden und in diesem Sinne so weit wie möglich in das Sozio der Linguistik (vgl. bereits Cameron 1990) vorzudringen. Im Kern geht es dieser Untersuchung also darum, soziale Zusammenhänge gerade aus einer linguistischen Perspektive zu durchleuchten, und damit vor allem auch linguistisch zu verstehen, wie denn die Mechanismen der Ungleichheit soziale, gar sprachlich-ethnische Gruppen und Schichtungen überhaupt erst konstruieren, bzw. auf welche Weise sich solche Schichtungen im Bildungswesen derart dauerhaft reproduzieren können, wie dies in modernen Migrationsgesellschaften eindrucksvoll der Fall ist (vgl. bereits Jungbluth 1994, S. 115; Rüesch 1998, S. 279; Kronig/Haeberlin/Eckhardt 2000, S. 42). Jedoch nicht allein die Reproduktion von Ungleichheit, sondern besonders auch das tiefere Verstehen ihres Gegenteils, des Durchbrechens einer dauerhaften sprachlich-ethnischen Schichtung, ist zentraler Gegenstand und Anliegen dieser soziolinguistischen Studie.

\section{Theoretischer Rahmen}

Um das traditionell soziologische mit einem linguistischen Verstehen sozialer Ungleicheit zu verbinden, wurde bereits für den theoretischen Rahmen zur vorliegenden Untersuchung nach verbindenden Elementen und Gemeinsamkeiten aus mehreren Disziplinen gesucht. Vier der für die Untersuchung identifizierten wichtigsten theoretisch-interdisziplinären Verbindungen werden im Folgenden ausgewählt und überblicksartig besprochen.

An erster Stelle ist, wenn es um soziale Ungleichheit und Bildung geht, ein Verständnis davon relevant, welche Rolle dabei die gesellschaftliche (hier: bildungspolitische) Ebene oder Makroebene in der Einwanderungsgesellschaft spielt, welches Gewicht ihr also im Vergleich zu anderen (z.B. institutionellen oder individuellen) Ebenen zukommt. Als bislang elaboriertestes, da auch empirisch erprobtes soziologisches Modell zum Zusammenspiel verschiedener Ebenen im Bildungserfolg gilt das ,Modell der segmentierten Assimilation“ aus einer US-amerikanischen Forschungsgruppe rund um Portes/Rumbaut (2001). Zusammenfassend besagt dieser Ansatz, dass die in der Migration erfolgreichsten Individuen ihren Erfolg gerade nicht aus dem Aufgeben, sondern aus dem Nutzbarmachen und Weiterführen von familiär bereits vorhandenen Sprachen und Beziehungen rekrutieren (ebd., S. 63 ff.). Das individuelle Nützen von Ressourcen erweist sich jedoch seinerseits auch als 
abhängig von den Bedingungen, die auf der gesellschaftlichen und damit „machtvolleren“" Ebene herrschen: „Our findings give a partial lie to the ,Where there is a will, there's a way " [...]) (Portes/Rumbaut 2001, S. 267).

Nicht auf ungleiche Bildung, wohl aber auf Ungleichheit und Spracherwerb und die Rolle der gesellschaftlichen (hier: sprachenpolitischen) Makroebene bezieht sich demgegenüber ein Modell aus einer ganz anderen, traditionell von der Soziologie weiter entfernten linguistischen Disziplin: aus der Forschung zu Language Shift. Als das elaborierteste Modell gilt hier das ,Gaelic Arvanitika Model' von Sasse (1992, S. 19; siehe auch Dressler/de Cillia 2006, S. 2260). Von besonderem Interesse ist dieses Modell aber nicht nur linguistisch, sondern - und hier liegt eine überraschende Verbindung - gerade auch soziologisch. Ganz ähnlich wie Portes/Rumbaut, so postuliert auch Sasse die an Auswirkungen überlegene Macht der gesellschaftlich-sprachenpolitischen Makroebene: Nur dieses „externe Setting“ kann genügend Druck erzeugen, um so weitreichende Folgen wie beispielsweise einen kollektiven Verlust von Sprachen und den Wechsel hin zu neuen, z.B. zu prestigereicheren Gemeinschaftssprachen auszulösen.

Ein erstes relevantes Theorieelement zum Thema soziale Ungleichheit verbindet also die soziologische Bildungsforschung mit der linguistischen Forschung zu Language Shift.

Als zweites Element, das für ein linguistisches Verständnis von Ungleichheit in der Migration relevant ist, erweist sich - neben der Makroebene der Einwanderungsgesellschaft - auch das der so genannten ,Herkunftsgesellschaft'. Überraschend zeigt sich in diesem Bereich, dass die Migration, und damit auch das angesprochene ,Herkunftsland' von einst immigrierten Familien, zwar oft schon länger zurückliegt, gleichwohl aber immer noch Spuren hinterlässt: Soziale (hier: bildungs- und sprachenpolitische) Ungleichbehandlung dürfte ein so machtvoller Faktor sein, dass sich ihre Wirkung selbst über Staatsgrenzen und Familiengenerationen hinweg lange erhält. Parallel entstandene theoretische Ansätze dazu gibt es mittlerweile sowohl in der Soziologie (Feliciano 2006, aus dem oben genannten Team um Portes/Rumbaut) als auch in der Soziolinguistik: Hier ließen sich an einem kleinen Untersuchungssample erste Verbindungen zwischen dem Herkunfts- und dem Einwanderungskontext ausmachen; diese führen von sprachenpolitisch bedingten Erfahrungen der Marginalisation und Sprachaufgabe im familiären Herkunftsland bis hin zu Phänomenen „kollektiver“ Ungleichheit des Spracherwerbs in der Migration (Sprachkapitalmodell, Brizić 2007, S. 173 ff.). Das zweite relevante Theorieelement resultiert also aus einer Parallele zwischen neuerer soziologischer und soziolinguistischer Migrations- und Bildungsforschung.

Dies führt direkt weiter zum dritten wesentlichen Element, einem Drehund Angelpunkt des Spracherwerbs: zur Weitergabe oder aber Aufgabe von angesammeltem Sprachkapital auf der familiären Mesoebene. Diese verbindet gesell- 
schaftliche Prozesse von Sprachen- und Bildungspolitik in Herkunfts- und Einwanderungsgesellschaft (Makroebene) mit dem individuellen Spracherwerb (Mikroebene) (Brizić 2007, orientiert u.a. an Bourdieu 1983). Auch dafür kommt die Grundlage aus der Soziologie (dort als, Intergenerationale Transmission' bezeichnet, vgl. Nauck/Diefenbach/Petri 1998); anders als dort haben wir es jedoch in unserem Zusammenhang vor allem mit Mechanismen der intergenerationalen Sprach-Transmission zu tun. Die Forschung zum Phänomen des Language Shift, auf der Ebene kollektiven Verlusts früher auch als „Sprachtod“-Forschung bezeichnet (Dressler/de Cillia 2006, S. 2259), gewinnt hier also erneut Bedeutung, wie schon vorhin auf der Makroebene.

Ganz klar sei hier jedoch gesagt, dass es in unserem Zusammenhang nicht etwa um Sprachwandel und -wechsel ,an sich“ geht, um jene mannigfaltigen Prozesse also, die in jeglicher Sprachverwendung unablässig stattfinden und die für die Flexibilität und Kreativität menschlichen Spracherwerbs stehen; und ebensowenig geht es hier um Anliegen des Spracherhalts. Vielmehr interessiert, dass und wie Language Shift sehr oft auch mit einem Gefälle an Macht und mit klar getrennten gesellschaftlichen Positionen verbunden sein kann, und solcherart mit böchst unterschiedlichen Bedingungen des Spracherwerbs, höchst unterschiedlichen Graden der „Freiwilligkeit“ dieses Wechselns von „alten“ zu „,neuen“ (Familien-)Sprachen (vgl. z.B. Wodak/ Rindler-Schjerve 1985 als eine der detailliertesten Studien zum kollektiven Sprachwechsel; vgl. demgegenüber Pennycook 1999 zur Diskussion unterschiedlicher Positionen bezüglich Sprachwechsel und Spracherhalt). Immerhin ist selbst unter den Bedingungen von Ungleichheit jeglicher Spracherwerb, Sprachwandel und -wechsel als kreativ bewältigbar einzuschätzen, und der Begriff einer vorgestellten, gar starren „Muttersprachlichkeit“ ist damit auch für den vorliegenden Zusammenhang lange überholt (vgl. Rampton 1995, S. 336). Auf Basis dieser grundlegenden Erkenntnisse also interessieren hier nur am Rande die vielen je kontextbedingten Formen des Wechselns zwischen den Sprachen, gerade in der Migration (vgl. z.B. Harris 2006; Rampton 2006, 2011); vielmehr soll es um die Frage gehen, was denn eigentlich - bei gleichzeitig grundsätzlicher Flexibilität des Sprachwechsels an sich - dennoch die verschiedenen, oft deutlichen Phänomene „sprachlicher Ungleichheit“ ausmacht (vgl. z.B. die eingangs genannte Literatur), verbunden mit der Frage, ob und wie sich etwas derart „Kollektives" wie soziale Ungleichheit denn auch in der familiären Sprachtransmission, in individuellen Sprachproduktionen und deren Beurteilung abbildet. Die Wirkungsmacht sozialer Ungleichheit würde es tatsächlich erwarten lassen, dass sie auf allen Ebenen Spuren hinterlässt; in diesem Zusammenhang kommt es auch nicht überraschend, dass beispielsweise in neueren Arbeiten zu Sprachkontakt und Sprachwandel zwischen dem ,gängigen“ 
kontaktbedingten Wandel einerseits und dem Wandel in „endangered languages“ andererseits klar unterschieden wird (vgl. Chamoreau/Léglise (Hg.) 2012).

Das dritte Theorieelement verbindet somit nicht nur Konzepte der soziologischen und soziolinguistischen Migrationsforschung, sondern beide wiederum mit Tendenzen und Konzepten aus der Forschung zu Sprachkontakt, Sprachwandel und Language Shift.

Wesentlich direkter als bei der Weitergabe von Sprachen ist die Wirkungsmacht sozialer Ungleichheit bei der Zuteilung von Bildungserfolg analysiert worden, also auf der schulisch-institutionellen Mesoebene (Portes/Rumbaut 2001; Gomolla/Radtke 2007 u.v.m.). Auch hier findet also eine Form der intergenerationalen Transmission statt, wenngleich nicht familiär, sondern zwischen Lehrkräften und Lernenden. Ein besonders detaillierter Blick auf die Mechanismen dieser „Transmission“ oder „Verteilung" von Bildungserfolg ist der Untersuchung von Gomolla/Radtke (2007) zum Funktionieren von Institutionen, im besonderen der Institution Schule, zu verdanken. Und es ist genau dieser institutionelle Aspekt, der auch für die hier besprochene soziolinguistische Untersuchung wesentlich und produktiv ist: Nicht nur ist ja die einzelne Schulklasse als Teil der Institution Scbule zu betrachten, sondern ebenso auch die einzelne Familie als Teil der "Institution“" (migrantische) Community.

Es verbindet diese vierte interdisziplinäre Übertragung von Konzepten also die (erziebungswissenschaftlich-soziologische) Bildungsforschung erneut mit der Forschung zu Language Shift, diesmal auf institutioneller Mesoebene.

Dabei sind alle genannten Ebenen jeweils zu verstehen als Orte nicht nur der Reproduktion von Ungleichbeit, sondern auch des potenziellen Durchbrechens und Veränderns von Ungleichheit, sei es sprachenpolitisch-institutionell (Makro-/ Mesoebene) oder auf der individuellen (Mikro-)Ebene der einzelnen Lehrkraft, des einzelnen Elternteils und des einzelnen Kindes.

\section{Samplebildung, Datensammlung und Analyse}

Wie die Theorie, so orientieren sich auch die Methoden der Samplebildung, der Datensammlung und der Analyse an der beschriebenen Grenzwanderung zwischen Sozio und Linguistik, und damit auch zwischen dem quantitativen und dem qualitativen Paradigma der Ungleichheitsforschung.

Das qualitative Kern-Sample besteht aus rund 180 Kindern. Diese Zahl ist einerseits groß genug, um für statistische Analysen gerade noch sinnvoll in Frage zu kommen, und andererseits klein genug, um gerade noch den Aufwand qualitativ-linguistischer Analysen zu erlauben. Die Kinder waren zum Zeitpunkt der Haupterhebung im Schnitt 10 Jahre alt und befanden sich am 
Übergang zwischen Grundschule und Sekundarstufe - ein Übergang, der in Österreich wie auch in anderen mitteleuropäischen Bildungssystemen für die gesamte weitere Bildungslaufbahn entscheidend ist, da ab diesem Zeitpunkt die Schülerpopulation auf die angesehenen (Gymnasium) und weniger angesehenen Schultypen (Hauptschule) ${ }^{5}$ aufgeteilt wird.

Insgesamt verteilten sich zur Zeit der Datenerhebung, also noch in der Grundschule, die Kinder des Samples auf 17 Schulen in zwei Wiener Bezirken mit hohem Migrantenanteil; die einzige Ausnahme bildete eine Gruppe von 13 Kindern im selben Alter, die nicht in Wien, sondern in Istanbul lebten bzw. leben, auch dort in einem migrantisch geprägten Stadtteil. ${ }^{6}$

Was die Migrationsgeschichte der rund 180 Familien betrifft, wurde versucht, einen möglichst weiten Bogen zwischen vielen unterschiedlichen Arten, Abschattierungen und Ausmaßen des so genannten ,Migrationshintergrunds' zu spannen: Während bei einem Teil der Kinder die familiäre Migration schon weit zurückliegt oder überhaupt nur einen sehr kleinen Teil der Familie betroffen hat, waren andere Kinder selbst erst kürzlich zugewandert; und während es sich in der Wiener Gruppe fast immer um eine Migration aus Gründen der Arbeitssuche handelt, oft kombiniert mit Familiennachzug, sind die Familien des Samples in Istanbul fast immer Flüchtlinge, die im Zuge bewaffneter politischer Konflikte ihr ursprüngliches Gebiet in der Osttürkei zu verlassen gezwungen wurden.

Wie bei der familiären Migrationsgeschichte, so wurde auch in Bezug auf die Mehrsprachigkeit eine breite Auffächerung des Samples angestrebt, von familiärer Einsprachigkeit über Zweisprachigkeit bis hin zu Vielsprachigkeit. Dabei wurde darauf geachtet, aus jeder der so genannten ,Herkunftsgruppen' (Herkunftsländer Türkei, ehemaliges Jugoslawien) jeweils sowohl schulisch „stark“ als auch „schwach“ abschneidende Kinder einzubeziehen. Die breite Streuung war also auch hier wieder von größter Bedeutung, um am Ende eventuell erkennbare Muster in möglichst zahlreichen qualitativen Schattierungen, gleichsam auf einem „Ungleichheits-Kontinuum“, nachvollziehen zu können.

Die qualitative Datenerhebung bezog von Anfang an sowohl die einzelnen Wiener Bezirksschulinspektionen als auch sämtliche Schuldirektionen, Lehrkräfte und Eltern der Kinder mit ein, allein schon wegen des aufwändigen Verfahrens des Einholens der Bewilligungen auf allen HierarchieEbenen. Dazu kamen im weiteren Verlauf noch eine Schulberatungsstelle und eine für die Sekundarstufe zuständige Schulbehörde.

Dieser kürzlich zur ,Neuen Mittelschule‘ umgewandelte österreichische Schultyp entspricht etwa der deutschen ,Realschule‘.

Unser Dank gilt hier den kooperierenden Personen an der Bilgi-Universität und dem Tarlabaşı Toplum Merkezi, beide in Istanbul. 
Die erhobenen Daten setzen sich somit zusammen aus:

1) „faktischen“ Informationen (Daten aus zwei offiziellen Bildungsdokumentationsquellen sowie aus von seiten der Schuldirektionen gegebenen Auskünften);

2) diskursiv gewonnenem Material (mehrfache Gespräche mit den Kindern sowie gemeinsam mit den Kindern ausgefüllte Fragebögen zu ihren Familiensprachen; umfangreiche narrativ-biografische Interviews mit den Eltern in Türkisch, Kurdisch, Armenisch, Bosnisch/Serbisch, Deutsch, teils auch Rumänisch und Romanes; narrative, teils auch biografische Interviews mit den Lehrkeäften, mit der Mitarbeiterin einer Beratungsstelle und dem Mitarbeiter einer schulbehördlichen Stelle, alle in Deutsch geführt);

3) schriftlichen Sprachproduktionen (Narrationen) der Kinder: ${ }^{7}$ Dieser Teil lag sowohl in seiner Konzeption und Erhebung als auch bezüglich Analyse in der Verantwortung der Dissertantin des Projekts und wird in ihrer Dissertation umfassend dargestellt ${ }^{8}$ (Blaschitz 2012); die Sprachen, in denen die Kinder Narrationen produzierten, sind Deutsch, Türkisch und Kurdisch sowie - allerdings nur mündlich - Romanes.

Ergänzt werden die genannten Datenquellen durch

4) Aufnahmen der mündlichen Nachbesprechungen mit dem Interviewteam und Aufbereitungen der umfangreichen Feldnotizen des gesamten Datenerhebungsteams.

Insgesamt sind es damit zwei rote Fäden, an denen sich die Datenerhebung zentral orientiert:

Erstens liegt das Schwergewicht explizit auf dem Diskurs, den diskursiv konstruierten, im Gespräch erinnerten, auch inszenierten Interpretationen und Bewertungen dessen, was die je eigene Sprach- und Bildungsbiografie sowie die des eigenen oder unterrichteten Kindes ausmacht und ausgemacht hat, Hemmnis oder Ressource bedeutet (hat). Es wurde diese Herangehensweise in enger Anlehnung an Gomolla/Radtke (2007) gewählt, deren Untersuchung die überragende Bedeutung der Interpretation, mehr als des „faktisch Geschehenen“, für den Bildungserfolg illustriert; es wurde deshalb auch im vorliegenden Projekt dem diskursiven Material der Vorzug gegenüber einem durch Beobachtung gewonnenen Material gegeben. Der Diskurs ist (auch) in unserem Fall immer institutionell konnotiert, selbst wenn z.B. die Interviews sehr oft bei den Familien daheim stattfanden, die Atmosphäre bei der Erhebung mit den Kindern möglichst locker gestaltet wurde und Ähnliches.

In zwei Durchgängen, wobei bislang nur der erste Durchgang in die engere Analyse miteinbezogen werden konnte.

8 Betreut von Konrad Ehlich, dem unser großer Dank gebührt. 
Zweitens liegt das Schwergewicht der Untersuchung auf der Narration, die daher im Material sowohl auf der (Mikro-)Ebene der Kinder als auch auf der familiären sowie schulischen (Meso-)Ebene der Eltern und Lehrkräfte eine zentrale Rolle spielt: Narrationen zu elizitieren war sowohl eines der Ziele in den Interviews, hier mit biografischem Kern," als auch das Ziel schlechthin in Bezug auf die Sprachproduktionen der Kinder. Der Grund dafür liegt nicht nur darin, dass die narrative Qualifizierung der Kinder einen zentralen Platz in der Sprachaneignung insgesamt (vgl. Ehlich 2007) wie auch im schulischen Kontext (Bredel 2001, S. 4) einnimmt, sondern vor allem darin, dass Narrationen auch in Ungleichheitskontexten eine Schlüsselrolle spielen dürften: In Situationen des Sprachwechsels unter sozial besonders schwierigen Bedingungen können Narrationen aus der ,alten“ Familiensprache oft nicht mehr in die neue Sprache mit übernommen werden (vgl. Wodak/Rindler-Schjerve 1985, S. 23-24), was als eine bedeutsame Einbuße in Bezug auf das kindliche Spracherwerbsmaterial betrachtet werden kann. - Beide Schwergewichte der qualitativen Erhebung, der Diskurs und die Narration, wurden in der Folge auch für die Analyse der qualitativen Daten beibehalten.

Die qualitative Analyse des gesammelten Materials ist derzeit (2012) in Gang, der Abschluss wird für Anfang 2013 erwartet. Die Analyse gliedert sich in zwei Hauptphasen:

Nach der Aufbereitung der Daten (d.h. Bereinigung, Transliteration, zu großen Teilen Transkription) wurde zunächst jener Projektteil abgeschlossen, der die Analyse der schriftlichen Narrationen der Kinder beinhaltete: Auf ihrer Grundlage wurden „narrative Profile“ als wesentliches Material für den Blick auf die (Mikro-)Ebene der Kinder erstellt (umfassend dargestellt in Blaschitz 2012).

Die erste Hauptphase der Gesamtanalyse, die hier anschließt, besteht nun darin, sämtliche Daten zu jedem Kind zusammenzuführen und die Kinder wiederum zu sozialen Gruppen zusammenzufassen, wobei diese Gruppen in verschiedenster Weise definiert werden: sprachlich, nach subjektiver „Zugehörigkeit", nach Herkunftsgebieten u.v.m.; dies geschieht jeweils in Relation zu dahinterliegenden gesellschaftlich-sozialen Hierarchien, wie etwa der bildungspolitischen Stellung einer Sprache in Herkunfts- und Einwanderungsgesellschaft. Der Kern der qualitativen Analyse fügt nun zu den gesammelten „Fakten“ (von Geburtsorten bis zu Schulnoten und Überweisungsempfehlungen) und kindlichen Sprachproduktionen (Narrationen) auch die „diskursiven" Inhalte (aus den Eltern- und Lehrerinterviews sowie aus den Gesprächen mit den Kindern, neben Feldnotizen) hinzu; diese werden nach diskursiven „Mustern“ durchleuchtet und zueinander in Relation gesetzt, um solcherart zu jenen Faktoren vorzudringen, die jeweils zu einer Repro-

Orientiert u.a. an Rosenthal (1995). 
duktion des ,gruppentypischen“ Abschneidens oder aber zu einem Durchbrechen der Reproduktion (,atypisches Abschneiden“) beigetragen haben könnten. Es geht hier also vor allem um eine diskursive Rekonstruktion horizontaler Verläufe, deren Widersprüche und deren Knackpunkte, an denen die Richtungen der einzelnen Bildungswege sich trennten und zu einem als ,erfolgreich“ oder „schwach“ kategorisierten Abschneiden führten. ${ }^{10}$

Die zweite Hauptphase der Gesamtanalyse geht hingegen vertikal vor, indem sie nun nicht mehr die einzelnen Kinder und ihre Verläufe, sondern die verschiedenen sozialen Gruppen zueinander in Beziehung setzt und auf jene Variablen hin vergleicht, die für den Bildungsverlauf von zentraler Bedeutung sein könnten.

Ein quantitatives Sample bildet zusätzlich den umfassenderen Hintergrund für die beschriebene qualitative Kerngruppe: In Anlehnung an eine niederländische quantitative Sprachenerhebung (Extra/Yağmur (Hg.) 2004) und in enger Kooperation mit dem niederländischen Team ${ }^{11}$ wurden im Rahmen unseres Projekts rund 20.000 Wiener Grundschulkinder der 3. und 4. Schulstufen mittels Fragebogen zu ihrem familiären und schulischen sprachlichen Alltag befragt. ${ }^{12}$ Auch das qualitative Sample, das hier nicht ganz ein Prozent ausmacht, ist selbstverständlich enthalten, um die qualitativen Ergebnisse in einen größeren Kontext zu stellen und zu überprüfen, ob und in welchem Ausmaß sich festgestellte „Muster“ und Zusammenhänge auch in den quantitativen Bereich hinein verfolgen lassen.

Insgesamt ist diese Form der Analyse dazu gedacht, Verbindungen in vielen verschiedenen Hinsichten herzustellen, soweit dies möglich ist: vom Mikroin den Makrobereich, von qualitativen Erklärungen zu quantitativen Daten und umgekehrt, vom schulischen zum familiären Diskurs, von großflächigen Phänomenen sozialer Ungleichheit bis zurück zur Sprachproduktion des einzelnen Kindes, und letztlich also von allen genannten Ausgangspunkten hin zu dem, was als ein „schwaches“ oder „starkes“ Abschneiden in der Institution Schule verstanden wird.

\section{Erste Einblicke in die Analysearbeit}

Für eine linguistische Ungleichheitsforschung sind gerade vielsprachige Bevölkerungsgruppen von größtem Interesse: Komplexer, und damit durchaus auch

10 Operationalisiert als die Überweisungsempfehlungen der Lehrkräfte sowie der Schultyp, an dem sich das Kind zwei Jahre nach der Hauptdatenerhebung befindet.

11 Unser Dank für das Zurverfügungstellen des Materials und für die enge Kooperation gilt Guus Extra und Karin Berkhout, Universität Tilburg, Niederlande.

12 Es beteiligten sich annähernd $90 \%$ der Wiener Grundschulen. Ein vorläufiger Bericht, für die Leserschaft der schulischen Praxis verfasst, ist bereits online: www.oeaw.ac.at/dinamlex/Multilin gual-Cities_Wien-2009_Endbericht-V1Stand20111111.pdf (Stand 04.06.2012). 
deutlicher als an anderen Gruppen manifestieren sich im Fall der Vielsprachigkeit vielfältige soziale Hierarchien, Bewertungs- und Abwertungsmechanismen gegenüber bestimmten Sprachen und sozialen Herkunftskonstellationen, die sich wiederum in familiären „Sprachenpolitiken “ und schulisch-institutionellen „Bildern“ niederschlagen. Es wurde dies im Übrigen bereits bei der Bildung unseres Samples wahrnehmbar: Die Kinder aus Familien mit mehr als zwei Alltagssprachen waren oft zugleich unter den schulisch als besonders „schwach“ eingeschätzten Kindern zu finden. Um die angesprochenen Zusammenhänge zu verdeutlichen, sollen hier zwei sehr gegensätzliche Beispielfälle in äußerster Kürze skizziert werden.

Das Kind SER, männlich und zur Zeit der Datenerhebung 10 Jahre alt, vereint in seinem familiären Hintergrund die Sprachen (bzw. Varietäten) Türkisch, Kurdisch-Zaza und Kurdisch-Kurmanci; dazu kommt aufgrund des Lebens in Österreich auch Deutsch. Die sprachliche Biografie der Familie von SER erfahren wir aus der Perspektive seiner Mutter, da sie es ist, die sich zum Interview zur Verfügung stellt.

Ihre Geschichte eröffnet die Mutter mit dem Beginn ihrer eigenen Schulzeit in der Osttürkei, einem im (außerschulischen, nichtinstitutionellen) Alltag dominant kurdischsprachigen Gebiet. Gleichzeitig führt die Mutter bereits ganz zu Beginn ihr Konzept des Begriffs der eigenen Sprache ein (siehe dazu Beispiel 1), einer Sprache, die familiär zunächst nicht weitergegeben wurde (da unsere Sprache verboten war, wie die Mutter vorangehend ausführt).

MU: Genelde bizimkiler sadece gizli bişey olduğu zaman kendi dilini konuşuyolardı.

odt:Bei uns haben meine Eltern nur, wenn sie etwas

Heimliches sprechen wollten, von ihrer eigenen sprache Gebrauch gemacht.

MU: Onun yüzünden ben kendi dilimi onsekiz yaşına kaç/ kadar hiç öğrenemedim.

odt:Aus diesem Grund habe ich meine eigene sprache bis zum achtzehnten Lebensjahr überhaupt nicht lernen können.

Die „eigene“ Sprache ist in diesem Konzept also bereits „eigen“ noch vor jedem Erwerbsprozess. Der Erwerb folgt im konkreten Fall erst später, und zwar als die Mutter in ihrer späteren Kindheit gerade durch die Aufforderung , sprich nicht (Kurdisch)!“" hellhörig wird und auf eigene Initiative die elterliche Sprache zu erlernen beginnt. In verstärktem Maß tut sie dies dann ab ihrer Migration nach Österreich, da hier Kurdisch-Zaza auch in Kursen 
angeboten wird, ebenso wie Kurdisch-Kurmanci, das durch die Heirat mit einem Mann aus derselben Herkunftsregion ebenfalls in die Familie Einzug hält. Die Geburt des Kindes SER schließlich erfordert die Wahl der Sprache(n) zwischen Eltern und Kind; diese wird von der Mutter als eine klare Entscheidung zugunsten von Kurdisch-Zaza dargestellt, relativiert allerdings durch die Umgebung, die meint, das Kind soll nicht durcheinander geraten, worauf die Mutter mehr Deutsch verwendet, sowie durch den Kinderarzt, der meint, das Kind solle besser die Muttersprache erlernen - womit er allerdings Türkisch meint -, worauf die Mutter mehr Türkisch verwendet.

Die Tendenz zur Verwendung von Kurdisch gewinnt dennoch im Lauf der Jahre wieder an Boden. Zur Zeit der Erhebung beherrscht das Kind SER außer Deutsch sowohl Türkisch als auch Kurdisch-Zaza. Letzteres verbessert SER in einem selbstgewählten Kurs; zudem hat das Kind auch vor, Kurdisch-Kurmanci zu lernen, und zeigt eine hohe Affinität zu beiden kurdischen Varietäten sowie zu Deutsch, weniger hingegen zu Türkisch. Das Türkische erfüllt hier u.a. die Funktion eines „mittleren Brückenpfeilers“, da es zwischen dem Kurdischen und dem Deutschen vermittelnd notwendig war und ist. Die Funktion des „Eigenen“ dagegen fällt dem Kurdischen zu, mit hoher Neigung auch zum Deutschen, das für das Kind als ein „,neues Eigenes“ bezeichnet werden könnte. Die sprachlich-lebensweltliche „Brücke“ des Kindes wird damit von den beiden „Eckpfeilern“ Kurdisch und Deutsch getragen.

Im linguistischen narrativen Profil in Deutsch (siehe weiter oben) schneidet das Kind sehr gut ab. Und auch die Lehrkraft des Kindes nimmt SER als außerordentlich erfolgreich und zudem als wissbegierig wahr; sie empfiehlt daher als Sekundarschultyp das Gymnasium, wo sich SER auch zum Zeitpunkt der Nacherhebung ${ }^{13}$ immer noch befindet.

Das Kind HÜS, ebenfalls männlich, gleich alt und aus ähnlichen sozioökonomischen Verhältnissen, unterscheidet sich gleichwohl sehr grundlegend von SER. Die Familie von HÜS ist aus der zentralanatolischen Türkei (Akdağmadeni, Provinz Yozgat) nach Österreich migriert und stammt damit ebenfalls aus einem mehrheitlich kurdischsprachigen, jedoch in tiefgreifenden Sprachwechselprozessen befindlichen Gebiet. Dem zentralanatolischen Kurdischen fehlt die Anbindung an die kompakten kurdischsprachigen Gebiete der Osttürkei; u.a. auch deshalb gewinnt das Türkische im Alltag an Raum. Hinzu kommt ein türkeispezifisches, historisch gewachsenes exorbitant niedriges Prestige nicht nur des Kurdischen insgesamt, sondern im Speziellen der zentralanatolischen Gebiete.

13 Rund zwei Jahre nach der Haupterhebung wurde überprüft, ob sich die Kinder (noch immer oder aber nicht mehr) in jenem Sekundarschultyp befinden, den die Lehrkraft am Ende der Grundschule empfohlen hatte. 
Der Vater von HÜS, mit dem das Interview geführt wird, hat familiär praktisch ausschließlich Kurdisch-Kurmanci gelernt; mit seinem Schuleintritt in der Türkei wird jedoch das Türkische fast übergangslos dominant. Mit der Heirat gewinnt für kurze Zeit das Kurdische nochmals an Gewicht, da die Ehefrau (und spätere Mutter von HÜS) kaum über Türkischkompetenz verfügt.

Das Kind HÜS, bereits in Österreich aufgewachsen, gibt übereinstimmend mit seinem Vater an, Kurdisch überhaupt nicht zu sprechen - und dies, obwohl Kurdisch die einzige Sprache ist, die die Mutter beherrscht. ${ }^{14}$ Die Mutter wird vom Vater etwas impliziter, von den Kindern dagegen explizit davon abgehalten, Kurdisch zu verwenden (Beispiel 2):

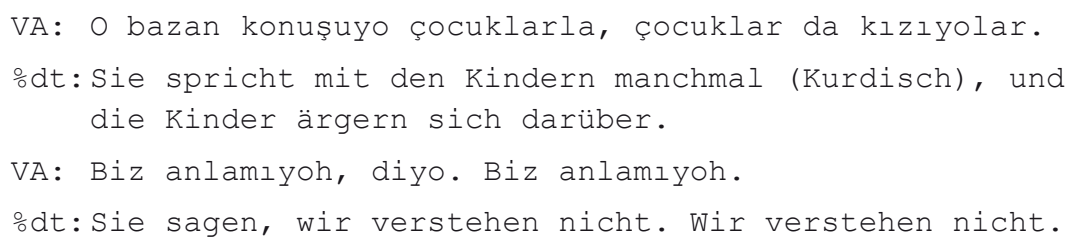

Dass HÜS und seine Geschwister nicht Kurdisch können, bedauert der Vater überhaupt nicht; dies mit der Begründung, dass gegenwärtig in der Türkei wabrlich nur Türkisch von Bedeutung ist. Wie hoch aber schätzt der Vater seine eigene Türkischkompetenz ein, anhand einer vom Interviewer beispielhaft gebotenen Skala von „sehr gut“ über „mittelmäßig“" bis „schlecht“ (Beispiel 3)?

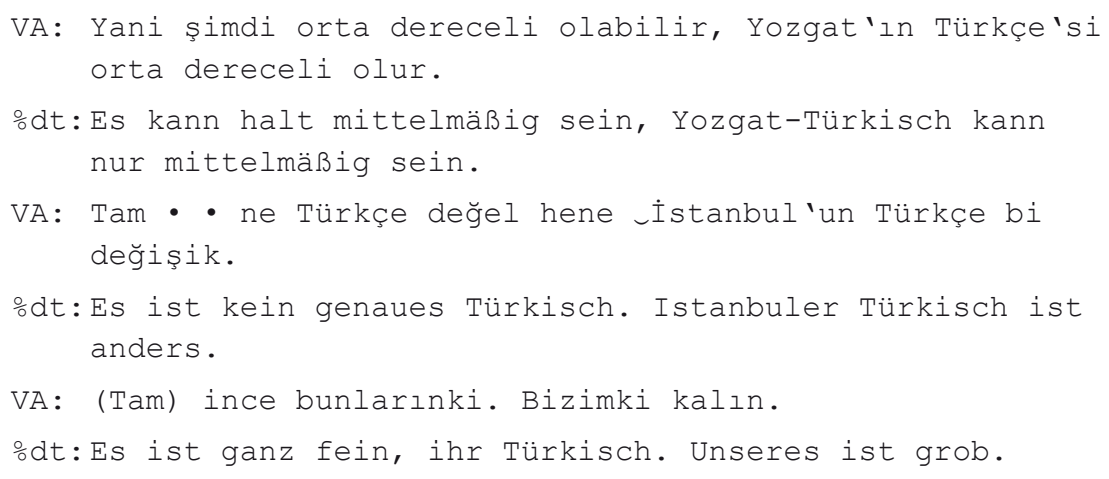

14 Da sie in ihrer Kindheit in der Türkei keine Schule besucht hatte, kam sie nur wenig in Kontakt mit dem Türkischen. 
Und in welcher Sprache besteht nun also die höchste Kompetenz (Beispiel 4)?

VA: Ya şu anda ben en iyi dil şu anda ... şu anda ben Avusturya 'da yaşadığım için,

odt:Na ja, derzeit, ich, die beste sprache, derzeit... da ich derzeit in Österreich lebe,

VA: bi Avusturya vatandaşı olduğum için, şu anda ben istiyorum ki keşke ben Almancam tam net olsa.

odt: österreichischer staatsbürger bin, wünsche ich mir, daß mein Deutsch einwandfrei wäre.

INT:Ama kendinizi en iyi hangi dilde ifade ediyorsunuz siz?

odt:Aber in welcher sprache drücken sie sich am besten aus?

VA: E şu anda ben Almanca'yı şu anda istiyorum.

odt: Ich will jetzt Deutsch, ich will die deutsche sprache.

INT: İstiyorsunuz ama, gerçekte?

odt:Sie wollen (es), aber in der Realität?

VA: Ha gerçekte tabi ki Türkçe. Türkçe ama, Almanca'yı istiyorum ben.

odt:Gut, in der Realität natürlich Türkisch. Türkisch, aber ich will Deutsch.

Die familiär vorgenommene Bewertung der drei beteiligten Sprachen Kurdisch, Türkisch und Deutsch - wird vom Vater in hoher Übereinstimmung mit dem Kind HÜS dargestellt: Das Kurdische versucht die Familie so vollständig wie möglich hinter sich zu lassen, selbst zum Preis einer hochgradig eingeschränkten Kommunikation mit der Mutter; das Türkische steht immerhin auf der Stufe einer Staatssprache, der es jedoch in Österreich an aktuellem Rang fehlt. Einzig Deutsch hat Bedeutung erhalten und gewinnt immer noch dazu.

Im narrativen Profil schneidet HÜS nur wenig schwächer als SER, jedoch in krassem Gegensatz zu seinem eigenen schulischen Erfolg ab: Das Kind befindet sich gar knapp an einer Sonderschulzuweisung. Von der Lehrkraft wird HÜS als extrem schwach, zugleich aber gerade auch als extrem beflissener Streber wahrgenommen (Beispiel 5) - 
LEHR: Er zerkreuzigt sich in Deutsch, er zerfuzelt sich.

- eine Darstellung also einer immensen Deutsch- und Aufstiegsmotivation des Kindes, die sich mit den Ausführungen des Vaters im Interview hochgradig deckt.

Insgesamt entsteht damit das Bild eines Kindes unter hohem Druck, das, ganz im Gegensatz zu SER, keine „Brücke“ zwischen ,alten“ und „,neuen“ Sprachen errichtet, sondern in stetigem „Gipfelsturm" begriffen scheint, weg von Kurdisch, weg von Türkisch, hin zu Deutsch.

Neben SER und HÜS finden sich in unserem Sample zahlreiche weitere Beispiele des Umgangs mit Vielsprachigkeit und der bedeutenden Rolle von „Übergangssprachen“, darunter genauer zu untersuchende Parallelen zwischen der kurdischen Gruppe einerseits (mit verschiedenen kurdischen Varietäten plus Türkisch, manchmal auch Arabisch, Tscherkessisch etc.) und der Minderheit der Roma andererseits (mit verschiedenen Varietäten des Romani plus Serbisch, manchmal auch Makedonisch, Albanisch, Rumänisch oder Türkisch). Die hier skizzierten Beispiele sind keinesfalls dazu angetan, die Komplexität des untersuchten Zusammenspiels zwischen Makro-, Mesound Mikroebene auch nur annähernd darzustellen oder einen Ausblick auf die Ergebnisse zu bieten. Vielmehr sind sie dazu gedacht, einen ersten Blick auf die Art der untersucbten Zusammenbänge zu werfen, und zwar exemplarisch anhand nur eines einzigen ausgewählten roten Fadens unter vielen, die sich voraussichtlich durch das umfangreiche Material hindurch verfolgen lassen.

\section{Abschließende Bemerkungen}

Das grundlegende Ziel der hier dargestellten Untersuchung ist das Identifizieren von Möglichkeiten, um aus neuen Perspektiven auf alte Phänomene zu schauen. Das alte Phänomen, die soziale Ungleichheit, menschengemacht und somit durch den Menschen veränderbar (Kreckel 1997, S. 13), birgt viel Potenzial für neue Perspektiven, wenn man bedenkt, wie wenig untersucht beispielsweise ein so verbreitetes Phänomen wie Language Shift im Kontext von Ungleichheit immer noch ist. Die neuen Perspektiven können aber nicht nur aus Phänomenen entstehen, die neu in Ungleichheitszusammenhängen betrachtet werden, sondern auch aus methodischen $\mathrm{He}-$ rangehensweisen, die neu in Ungleichheitszusammenhängen angewendet werden. Ein möglichst sensibles Ausloten der Verbindungen und Grenzen zwischen der meist quantitativen Bildungsforschung und der qualitativen (Sozio-)Linguistik bleibt deshalb die vielleicht größte Herausforderung dieser Untersuchung. 
Der qualitative Zugang mag dennoch bisher die Oberhand behalten haben: Das Erübrigen von Zeit, um Befragte ausführlich zu Wort kommen zu lassen, hat die Datenerhebung geprägt und wird auch die Analyse des umfangreichen Materials ausmachen. Wie sonst könnten so komplexe und zutiefst persönliche Informationen wie die Weitergabe und der Verlust von Familiensprachen (bei den Eltern) oder aber die Weitergabe und der Verlust von Bildungszielen (bei den Lehrkräften) überhaupt angesprochen, diskursiv ausgebreitet und - vielleicht - verstanden werden? Und wie könnte die Bedeutung, die Menschen diesen und vielen anderen Aspekten geben, eruiert werden, wenn das extensive Zu-Wort-Kommen, im Schulalltag ohnehin zu selten realisiert, auch in einer Untersuchung schließlich fehlt?

Das empirisch möglichst sensible Zuhören steht in enger Verbindung mit einer auch theoretisch „sensibilisierenden“ Herangehensweise (Denzin 1970, S. 14 f.), also einer möglichst großen Offenheit besonders für neue Konzepte, um solcherart zu einem Bilden von Theorie zu gelangen, das der interdisziplinären Herangehensweise dieser Untersuchung am ehesten entspricht (vgl. Brannen 1992, S. 4 ff.).

Dass jedoch, bei solch qualitativen Herangehensweisen, die Einbettung in soziale Zusammenhänge allzu oft fehle, wurde der Linguistik einst vorgeworfen (Esser 2006, S. 21). Die begonnene Untersuchung versucht, diese Kritik ernst zu nehmen.

\section{Literatur}

Blaschitz, Verena (2012): Dimensionen der Erfassung narrativer Qualifizierung bei Zehnjährigen mit Deutsch als Zweitsprache. 2 Bde. Dissertation. Wien.

Bourdieu, Pierre (1983): Ökonomisches Kapital, kulturelles Kapital, soziales Kapital. In: Kreckel, Reinhard (Hg.): Soziale Ungleichheiten. (= Soziale Welt; Sonderbd. 2). Göttingen, S. 183-198.

Brannen, Julia (1992): Combining qualitative and quantitative approaches: an overview. In: Brannen, Julia (Hg.): Mixing methods: Qualitative and quantitative research. Aldershot u.a., S. 3-37.

Bredel, Ursula (2001): Ohne Worte: Zum Verhältnis von Grammatik und Textproduktion am Beispiel des Erzählens von Bildergeschichten. In: Didaktik Deutsch 11, S. 4-21.

Brizić, Katharina (2009): Ressource Familiensprache. Eine soziolinguistische Untersuchung zum Bildungserfolg in der Migration. In: Schramm, Karen/Schroeder, Christoph (Hg.): Empirische Zugänge zu Sprachförderung und Spracherwerb in Deutsch als Zweitsprache. (= Mehrsprachigkeit 23). Münster, S. 23-42.

Brizić, Katharina (2007): Das geheime Leben der Sprachen. Gesprochene und verschwiegene Sprachen und ihr Einfluss auf den Spracherwerb in der Migration. (= Internationale Hochschulschriften 465). Münster u.a. 
Cameron, Deborah (1990): Demythologizing sociolinguistics: why language does not reflect society. In: Joseph, John Earl/Taylor, Talbot J. (Hg.): Ideologies of language. (= Routledge Politics of Language Series). London u.a., S. 79-93.

Chamoreau, Claudine/Léglise, Isabelle (Hg.) (2012): Dynamics of contact-induced language change. (= Language Contact and Bilingualism 2). Berlin/New York.

Denzin, Norman K. (1970): The research act in sociology. A theoretical introduction to sociological methods. 2. Aufl. London.

Dressler, Wolfgang U./de Cillia, Rudolf (2006): Spracherhaltung, Sprachverfall, Sprachtod. Language maintenance, language decline and language death. In: Ammon, Ulrich et al. (Hg.): Sociolinguistics. An international handbook of the science of language and society/Soziolinguistik. Ein internationales Handbuch zur Wissenschaft von Sprache und Gesellschaft. Bd. 2. 2., neu bearb. Auflage. (= Handbücher zur Sprach- und Kommunikationswissenschaft 3.2). Berlin/New York, S. 22582271.

Ehlich, Konrad (2007): Sprachaneignung und deren Feststellung bei Kindern mit und ohne Migrationshintergrund: Was man weiß, was man braucht, was man erwarten kann. In: Ehlich, Konrad (Hg.): Anforderungen an Verfahren der regelmäßigen Sprachstandsfeststellung als Grundlage für die frühe und individuelle Förderung von Kindern mit und ohne Migrationshintergrund. (= Bildungsreform 11). Berlin, S. 11-77.

Esser, Hartmut (2006): Sprache und Integration. Die sozialen Bedingungen und Folgen des Spracherwerbs von Migranten. Frankfurt a.M u.a.

Extra, Guus/Yağmur, Kutlay (Hg.) (2004): Urban multilingualism in Europe: immigrant minority languages at home and school. (= Multilingual Matters Series 130). Clevedon u.a.

Feliciano, Cynthia (2006): Beyond the family: the influence of premigration group status on the educational expectations of immigrants' children. In: Sociology of Education 79, S. 281-303.

Gomolla, Mechthid/Radtke, Frank-Olaf (2007): Institutionelle Diskriminierung. Die Herstellung ethnischer Differenz in der Schule. 2., durchges. u. erw. Aufl. Wiesbaden.

Haque, Zubaida (2000): The ethnic minority „underachieving“ group? Investigating the claims of ,underachievement" amongst Bangladeshi pupils in British secondary schools. In: Race, Ethnicity and Education 3, 2, S. 145-168.

Harris, Roxy (2006): New ethnicities and language use. (= Language and Globalization). Basingstoke u.a.

Herzog-Punzenberger, Barbara (2003): Ethnic segmentation in school and labour market? 40 years legacy of Austrian guestworker policy. In: Crul, Maurice/Vermeulen, Hans (Hg.): The future of the second generation. The integration of migrant youth in six European countries. (= International Migration Review 37,4/144). New York, S. $1120-1124$.

Hradil, Stefan/Schiener, Jürgen (2001): Soziale Ungleichheit in Deutschland. 8., überarb. u. aktual. Aufl. Opladen. 
Jungbluth, Paul (1994): Lehrererwartungen und Ethnizität. Innerschulische Chancendeterminanten bei Migrantenschülern in den Niederlanden. In: Zeitschrift für Pädagogik 40, S. 113-125.

Kreckel, Reinhard (1997): Politische Soziologie der sozialen Ungleichheit. (= Theorie und Gesellschaft 25). Frankfurt a.M./New York.

Kronig, Winfried/Haeberlin, Urs/Eckhart, Michael (2000): Immigrantenkinder und schulische Selektion. Pädagogische Visionen, theoretische Erklärungen und empirische Untersuchungen zur Wirkung integrierender und separierender Schulformen in den Grundschuljahren. (= Beiträge zur Heil- und Sonderpädagogik 26). Bern.

Maas, Utz/Mehlem, Ulrich (2002): Schriftkulturelle Probleme der Migration: Kinder marokkanischer Einwanderer in Deutschland. In: Oltmer, Jochen (Hg.): Migrationsforschung und Interkulturelle Studien. Zehn Jahre IMIS. (= Schriften des Instituts für Migrationsforschung und Interkulturelle Studien 11). Osnabrück, S. 333-366.

Nauck, Bernhard/Diefenbach, Heike/Petri, Kornelia (1998): Intergenerationale Transmission von kulturellem Kapital unter Migrationsbedingungen. Zum Bildungserfolg von Kindern und Jugendlichen aus Migrantenfamilien in Deutschland. In: Zeitschrift für Pädagogik 44, S. 701-722.

Peltzer-Karpf, Annemarie et al. (2006): „A kući sprecham Deutsch.“ Sprachstandserhebung in multikulturellen Volksschulklassen. Wien.

Pennycook, Alastair (1999): Development, culture and language: ethical concerns in a postcolonial world. In: Shaw, Jonathan/Lubelska, Diana/Noullet, Michelle (Hg.): Proceedings of the Fourth International Conference on Language and Development. Internet: www.languages.ait.ac.th/hanoi_proceedings/contents.htm (Stand: 08.09.2008).

Portes, Alejandro/Rumbaut, Rubén G. (2001): Legacies: the story of the immigrant second generation. Berkeley/London.

Rampton, Ben (1995): Crossing. Language and ethnicity among adolescents. (= Real Language Series). London u.a.

Rampton, Ben (2006): Language in late modernity: interaction in an urban school. (= Studies in Interactional Sociolinguistics 22). Cambridge u.a.

Rampton, Ben (2011): From ,multi-ethnic adolescent heteroglossia' to ,contemporary urban vernaculars'. In: Language \& Communication 31, S. 276-294.

Rosenthal, Gabriele (1995): Erlebte und erzählte Lebensgeschichte. Gestalt und Struktur biographischer Selbstbeschreibungen. Frankfurt a.M. u.a.

Rüesch, Peter (1998): Spielt die Schule eine Rolle? Schulische Bedingungen ungleicher Bildungschancen von Immigrantenkindern - eine Mehrebenenanalyse. (= Explorationen 18). Bern u.a.

Sasse, Hans-Jürgen (1992): Theory of language death. In: Brenzinger, Matthias (Hg.): Language death. Factual and theoretical explorations with special reference to East Africa. (= Contributions to the Sociology of Language 64). Berlin/New York, S. 7-30. 
Söhn, Janina/Özcan, Veysel (2006): The educational attainment of turkish migrants in Germany. In: Turkish Studies 7, S. 101-124.

Stanat, Petra (2003): Migration und Sozialschicht. Determinanten der Schulleistungen von Jugendlichen aus zugewanderten Familien. Unveröffentlichtes Manuskript zum Vortrag, gehalten bei der 64. Tagung der AEPF (Arbeitsgruppe für Empirische Pädagogische Forschung), 29.09.-02.10.2003, Universität Hamburg. Hamburg.

Tilmatine, Mohamed (1997): L'enseignement des langues maternelles en Europe: la fin d'une mystification? In: Tilmatine, Mohamed (Hg.): Enseignement des langues d'origine et immigration nord-africaine en Europe: langue maternelle ou langue d'etat? Paris, S. 69-101.

Weiss, Hilde et al. (2007): Leben in zwei Welten. Zur sozialen Integration ausländischer Jugendlicher der zweiten Generation. Wiesbaden.

Wodak, Ruth/Rindler-Schjerve, Rosita (1985): Funktionen der Mutter beim Sprachwechsel: Konsequenz für die Primärsozialisation und Identitätsentwicklung. Wiesbaden. 